\title{
Development and characterization of fifteen polymorphic microsatellite loci in Bryconamericus aff. iheringii (Teleostei: Characidae) and cross-amplification in related Characidae species
}

\author{
Caroline Apolinário-Silva ${ }^{1}$, Dhiego G. Ferreira ${ }^{2}$, Analiza F. Cavenagh ${ }^{2}$, \\ Nícollas G. O. Aprígio ${ }^{2}$, Bruno A. Galindo ${ }^{2}$, Jens Carlsson ${ }^{3}$ and Silvia H. Sofia ${ }^{1}$
}

Data on 15 novel microsatellite loci from the Neotropical fish Bryconamericus aff. iheringii are presented here. Analyses of 32 individuals from four different streams revealed 192 different alleles, ranging from four to 32 alleles per locus (mean of 12.8 per locus). Observed and expected heterozygosities ranged from 0.094 to 0.813 and 0.205 to 0.952 , respectively. These loci showed high polymorphic information content and will be a resource for genetic studies of $B$. aff. iheringii. Furthermore, several loci also amplified other small Neotropical Characidae (Piabarchus stramineus and Piabina argentea) and should be useful for these species.

Keywords: Genetic diversity, Lambarizinho, Molecular markers, Neotropical fish, SSR.

Um total de quinze novos locos microssatélites é aqui apresentado para o pequeno peixe Neotropical Bryconamericus aff. iheringii. A análise de 32 indivíduos provenientes de quatro ribeirões diferentes revelou 192 alelos diferentes, variando de quatro a 32 alelos por loco (média de 12,8 por loco), e heterozigozidades observada e esperada variando de 0,094 a 0,813 e 0,205 a 0,952 , respectivamente. O conjunto de locos obtido mostrou alto conteúdo de informação polimórfica e bom potencial para estudos genéticos de $B$. aff. iheringii, além disso vários locos amplificaram para outras espécies de pequenos Characidae neotropicais (Piabarchus stramineus and Piabina argentea).

Palavras-chave: Diversidade genética, Lambarizinho, Marcadores moleculares, Peixe Neotropical, SSR.

\section{Introduction}

Bryconamericus Eigenmann, 1907 is one of the most diverse genera of the Characidae family, occurring in many river systems in of the Neotropical region, from Costa Rica to Argentina (Vari, Siebert, 1990; Lima et al., 2003). They are small omnivorous fish (not exceeding $10 \mathrm{~cm}$ in length) and inhabit a range of environments, including streams, rivers and lakes (Britski et al., 1988). Species of this genus are important food sources for piscivorous fish (Britski et al., 1988) throughout the range of environments, and in the Paraná River basin (one of the main drainage systems in South America) the genus is a prey item of several piscivorous fish (Hahn et al., 1997; Lowe-McConnell, 1999).
Bryconamericus aff. iheringii (Boulenger, 1887), regionally known as pequira or lambarizinho, is a member of this genus distributed in the upper reaches of the Paraná River basin (Graça, Pavanelli, 2007). Studies have suggested that $B$. aff. iheringii from the Paraná basin may constitute a distinct species from the $B$. iheringii found in the Laguna dos Patos system, Southern Brazil (Langeani et al., 2005, 2007). However, $B$. aff. iheringii populations are commonly found throughout the upper Paraná sub-basins, including rivers and small streams (Langeani et al., 2007; Costa et al., 2013; Cetra et al., 2016).

Fish less than $150 \mathrm{~mm}$, such as $B$. aff. iheringii (maximum total length of $60 \mathrm{~mm}$, Graça, Pavanelli, 2007), often dominate the ichthyofauna of Neotropical streams (Castro,

${ }^{1}$ Laboratório de Genética e Ecologia Animal (LAGEA), Departamento de Biologia Geral, Universidade Estadual de Londrina, Rod. Celso Garcia Cid, km 380, 86051-970 Londrina, PR, Brazil. (CAS) carolapolinario07@gmail.com, (SHS) shsofiabelh@gmail.com, (Dhttp://orcid.org/0000-0002-3443-0696 (corresponding author)

${ }^{2}$ Laboratório de Genética e Conservação (GECON)², Universidade Estadual do Norte Paraná, Rua Portugal, 340 86300-000 Cornélio Procópio, PR, Brazil. (DGF) dhiegouenp@gmail.com, (AFC) chatcavenagh@hotmail.com, (NGOA) aprigio.bio@hotmail.com, (BAG) bruno@uenp.edu.br

${ }^{3}$ School of Biology and Environmental Science, UCD Science Education and Research Centre - West, University College Dublin Dublin 4, Ireland. jens.carlsson@ucd.ie 
1999; Winemiller et al., 2008). The high prevalence of small fish dominating the ichthyofauna raises questions about the evolutionary and population aspects of these species. According to Castro (1999), the small size of these species usually contributes to a low movement capacity along the drainages. This reduced movement capacity could be one of the underlying explanations for the many endemic species and small-scale population structure found in Neotropical areas (Castro et al., 2003; Ferreira et al., 2016). However, genetic data for these species are largely lacking (Sofia et al., 2006, 2008; Ferreira et al., 2016). Biological data including genetic analyses of small fish, such as Bryconamericus spp., are essential for understanding biological and evolutionary properties, as well as for the management and conservation of the ichthyofauna of Neotropical streams (Agostinho et al., 2005; Dudgeon et al., 2006). Microsatellites are highly variable genetic markers suitable for cost-effective population genetic studies. However, particularly in the case of Neotropical fish species, the lack of suitable genetic markers has been one of the main obstacles to the development of more complete genetic studies involving this diverse group of fish. Thus, the present study aimed to identify and develop polymorphic microsatellite loci for Bryconamericus aff. iheringii and assess cross-species transferability to four other small Characidae from the Neotropical region.

\section{Material and Methods}

Microsatellite loci were obtained from an enriched genomic library using the methods described by Billotte et al. (1999) with some minor modifications (cf. Ferreira et al., 2013). The required genomic DNA was extracted from a sample of Bryconamericus aff. iheringii using the method of Almeida et al. (2001). Briefly, $5 \mu \mathrm{g}$ was digested using $50 \mathrm{U}$ of RsaI endonuclease and specific adapters RsaI21 (5'CTCTTGCTTACGCGTGGACTA3') and RsaI-25 (5'TAGTCCACGCGTAAGCAAGAGCACA3') were linked to digested DNA using $5 \mathrm{U}$ of T4 DNA ligase (Invitrogen; www.invitrogen.com) in reaction buffer, including $10 \mu \mathrm{M}$ of each adapter in a final volume of $200 \mu \mathrm{l}$ and incubated at $20^{\circ} \mathrm{C}$ for $2 \mathrm{~h}$. Fragments with putative microsatellite sequences were obtained using three probes bound to biotin: $(\mathrm{AGA})_{5},(\mathrm{CT})_{8}$ and $(\mathrm{GT})_{8}$. These fragments were amplified by Polymerase Chain Reaction and cloned into pGem-T Easy (Promega; www.promega.com) vectors using $5 \mu$ l of amplification product, $50 \mathrm{ng}$ of vector and $1 \mathrm{U}$ of T4 DNA ligase in reaction buffer at $4^{\circ} \mathrm{C}$ (overnight). Cloning products were used to transform Escherichia coli (DH5- $\alpha$ lineage) cells.

A total of 96 positive clones were amplified using M13 primers ( $\mathrm{F}$ and $\mathrm{R}$ ) and subsequently sequenced using a Big Dye Terminator 3.1 sequencing kit (Applied Biosystems; www.appliedbiosystems.com) on an automated sequencer (ABI3500 xL, Applied Biosystems). BioEdit v.7.0 software (Hall, 1999) was used to visualize sequences and the Primer3 program (Rozen, Skaletsky, 2000) to generate microsatellite locus specific primers. Tests for potential hairpin structures and primer-dimers were conducted in AutoDimer software (Vallone, Butler, 2004).

Microsatellite validation was performed on a panel consisting of 32 individuals of $B$. aff. iheringii sampled from four streams (eight individuals per stream) in the Cinzas

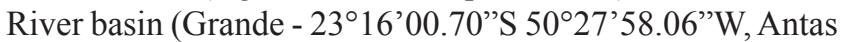

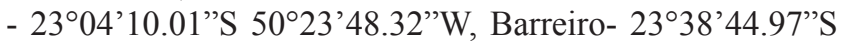
$50^{\circ} 23^{\prime} 32.32^{\prime \prime} \mathrm{W}$, and Bonito - $23^{\circ} 23^{\prime} 42.90^{\prime \prime} \mathrm{S} 50^{\circ} 10^{\prime} 8.61^{\prime \prime} \mathrm{W}$ streams), Southern Brazil. Cross-species amplification was trailed on extracted DNA from four other small Characidae species (five individuals per species), Piabarchus stramineus (Eigenmann, 1908), Piabina argentea Reinhardt, 1867, Serrapinnus notomelas (Eigenmann, 1915), and Hyphessobrycon eques (Steindachner, 1882), captured in the same drainages. Voucher specimens were deposited in the Museu de Zoologia da Universidade Estadual de Londrina (MZUEL) fish collection under catalog numbers, as follows: MZUEL 6457 (Bryconamericus aff. iheringii), MZUEL 6460 (Piabarchus stramineus), MZUEL 6466 (Piabina argentea), MZUEL 6467 (Serrapinnus notomelas) and MZUEL 6468 (Hyphessobrycon eques).

Microsatellite fragment length polymorphisms were analyzed on an automatic sequencer ABI PRISM $3500 \mathrm{xL}$, using GeneScan $600 \mathrm{Liz}$ (Applied Biosystems) as standard molecular weight. Fluorescent labeling of fragments followed the method of Schuelke (2000), which includes an additional M13 sequence (5'- TGTAAAACGACGGCCAGT-3') on the 5 ' end of the forward primer.

PCR reactions were conducted in a total volume of $5 \mu$, including $1 \mathrm{X}$ GoTaq Master Mix (Promega), $10 \mathrm{ng}$ of template DNA, $1 \%$ glycerol, $0.125 \mu \mathrm{M}$ labeled M13 primer (either FAM, HEX or NED, Applied Biosystems, CA), $0.125 \mu \mathrm{M}$ reverse primer, and $0.0125 \mu \mathrm{M}$ forward primer. Amplifications were performed with an initial denaturation step at $94{ }^{\circ} \mathrm{C}$ for $4 \mathrm{~min}$, followed by 35 cycles at $94^{\circ} \mathrm{C}$ for $40 \mathrm{~s}, 48^{\circ} \mathrm{C}, 52^{\circ} \mathrm{C}, 56^{\circ} \mathrm{C}$, or $60^{\circ} \mathrm{C}$ for $1 \mathrm{~min}, 72^{\circ} \mathrm{C}$ for $1 \mathrm{~min}$, and a final extension at $72^{\circ} \mathrm{C}$ for $30 \mathrm{~min}$.

Genotyping was performed using the GeneMarkerv.1.85 program (SoftGenetics; www.softgenetics.com). Cervus v.3.0 (Marshall et al., 1998) was used to calculate the number of alleles $(k)$, observed $\left(H_{\mathrm{o}}\right)$ and expected $\left(H_{\mathrm{e}}\right)$ heterozygosities, and polymorphic information content (PIC). The software package GENEPOP 4.0.10 (Raymond, Rousset, 1995; Rousset, 2008, using default settings) was used to test for conformance to the Hardy-Weinberg equilibrium (HWE) and linkage disequilibrium (LD) and resulting probability values were corrected for multiple tests using the sequential Bonferroni correction (Rice, 1989). The genetic identity (I) and paternity exclusion $(Q)$ probabilities (Paetkau et al., 1995; Weir, 1996) were estimated using Identity 1.0 (Wagner, Sefc, 1999, default settings). Endogamy coefficient $\left(F_{\text {IS }}\right)$ was estimated using the program Arlequin 3.5 (Excoffier, Lischer, 2010). Tests (default settings) for the presence of null alleles and genotyping errors caused by large allele dropout and stutter were assessed using the programme MicroChecker v.2.2.3 (Van Oosterhout et al., 2004). 


\section{Results}

Analyses of chromatograms for the 96 clones confirmed the presence of microsatellite repeats in a total of 46 sequenced clones. Based on sequence quality, microsatellite repeat structure, and primer suitability 15 loci (almost all dinucleotide repeats) were selected for further testing. These 15 loci all amplified successfully, showing polymorphisms that could be genotyped unambiguously. Information for these loci was submitted to the GenBank (accession numbers: MF693815MF693829, Tab. 1).

A total of 192 alleles were detected across the 15 microsatellite loci (mean of 12.8 per locus), ranging from four (Bih26) to 32 (Bih16) alleles per locus (Tab. 1).
The Bih42 locus showed the lowest observed $\left(H_{\mathrm{o}}=0.094\right)$ and expected $\left(H_{\mathrm{e}}=0.205\right)$ heterozygosities while the Bih16 showed the highest observed and expected heterozygosity $\left(H_{\mathrm{o}}\right.$ $=0.813$ and $H_{\mathrm{e}}=0.952$, respectively). The mean Polymorphic Information Content (PIC) for the 15 loci was 0.686. Following a scale proposed by Botstein et al. (1980), 12 loci (Bih13, Bih16, Bih19, Bih22, Bih30, Bih31, Bih40, Bih44, Bih45, Bih47, Bih76, and Bih77) were highly informative, with PIC values $>0.5$, two loci $(B i h 26$ and Bih73) were moderately informative (PIC $>0.25$ and $<0.5$ ), and only Bih42 showed low informative potential, PIC $<0.25$ (Tab. 1). This set of loci demonstrated a very low value of genetic identity combined probability $\left(4.7 \times 10^{-6}\right)$ and high combined probabilities of paternity exclusion (0.999), indicating high discriminatory power for population genetic studies.

Tab. 1. Description of 15 polymorphic microsatellite loci isolated from Neotropical fish Bryconamericus aff. iheringii. Ta optimal annealing temperatures; $k$, number of alleles; allele size range (bp); $H_{\mathrm{o}}$, observed heterozygosity; $H_{\mathrm{e}}$, expected heterozygosity estimated from 32 individuals; PIC, polymorphic information content; $Q$, paternity exclusion probability; $I$, probability of genetic identity; $F_{\text {IS }}$, endogamy coefficient; GenBank accession numbers. *Significant value of endogamy coefficient $\left(F_{\text {IS }}\right)$.

\begin{tabular}{|c|c|c|c|c|c|c|c|c|c|c|c|c|}
\hline $\begin{array}{l}\text { Locus } \\
\text { name }\end{array}$ & Sequence repeat & Primer sequences $\left(5^{\prime}-3^{\prime}\right)$ & $\begin{array}{c}\mathrm{Ta} \\
\left({ }^{\circ} \mathrm{C}\right)\end{array}$ & $k$ & $\begin{array}{l}\text { Allele size } \\
\text { range (bp) }\end{array}$ & $H_{\mathrm{o}}$ & $H_{\mathrm{e}}$ & PIC & $(Q)$ & $(I)$ & $F_{\text {IS }}$ & $\begin{array}{l}\text { GenBank } \\
\text { accession }\end{array}$ \\
\hline Bih13 & $(\mathrm{CA})_{14}$ & $\begin{array}{l}\text { F: CGCTCATTCAAGGTGAGAGTC } \\
\text { R: CTACGCCTTCCTACGCTGAG }\end{array}$ & 56 & 22 & $184-258$ & 0.719 & 0.933 & 0.929 & 0.864 & 0.285 & $0.244^{*}$ & MF693815 \\
\hline Bih16 & $(\mathrm{GT})_{17}$ & $\begin{array}{l}\text { F: TAAGGAGAGCGTCAGAATCC } \\
\text { R: CAGCAGCTCAGCTCTTCATC }\end{array}$ & 56 & 32 & $201-351$ & 0.813 & 0.952 & 0.950 & 0.903 & 0.275 & $0.162 *$ & MF693816 \\
\hline Bih19 & $(\mathrm{AC})_{14}$ & $\begin{array}{l}\text { F: GGCATAAGGAATCCAGCATATC } \\
\text { R: GTAACCGCTGATCACTAATAAGG }\end{array}$ & 56 & 24 & $169-243$ & 0.781 & 0.930 & 0.926 & 0.858 & 0.287 & $0.175^{*}$ & MF693817 \\
\hline Bih22 & $(\mathrm{AG})_{13} \mathrm{GG}(\mathrm{AG})_{3}$ & $\begin{array}{l}\text { F: GGTCCTGCACTCGCTGTTAG } \\
\text { R: GCCAGCCAACGGTTAGAG }\end{array}$ & 52 & 19 & $211-281$ & 0.375 & 0.853 & 0.840 & 0.852 & 0.336 & $0.570 *$ & MF693818 \\
\hline Bih26 & $(\mathrm{GT})_{4} \mathrm{~A}(\mathrm{AG})_{5}$ & $\begin{array}{l}\text { F: GAACTGCACGTTACATTCAGGA } \\
\text { R: CTCAGCACTTCTGCCTGAC }\end{array}$ & 52 & 4 & $175-197$ & 0.250 & 0.514 & 0.465 & 0.285 & 0.658 & $0.524 *$ & MF693819 \\
\hline Bih30 & $(\mathrm{TG})_{14}$ & $\begin{array}{l}\text { F: CTCCACGGCTGAAACAATG } \\
\text { R: CCAGCGAATAATCAGTCTTCC }\end{array}$ & 48 & 7 & $159-187$ & 0.414 & 0.601 & 0.551 & 0.440 & 0.493 & $0.327^{*}$ & MF693820 \\
\hline Bih31 & $(\mathrm{GT})_{14}$ & $\begin{array}{l}\text { F: GCATTGATATCCTGCCATCC } \\
\text { R: TCTACCTGTCCATCGTGTCC }\end{array}$ & 52 & 19 & $182-260$ & 0.310 & 0.893 & 0.885 & 0.903 & 0.303 & $0.662 *$ & MF693821 \\
\hline Bih40 & $(\mathrm{TG})_{2} \mathrm{C}(\mathrm{TG})_{12}$ & $\begin{array}{l}\text { F: ACAGCGCATTCCTCCATTC } \\
\text { R: AATCGACACGCTGCCAAG }\end{array}$ & 48 & 12 & $201-235$ & 0.500 & 0.712 & 0.686 & 0.516 & 0.449 & $0.312 *$ & MF693822 \\
\hline Bih42 & $(\mathrm{AC})_{14} \mathrm{AA}(\mathrm{AC})_{3}$ & $\begin{array}{l}\text { F: GAGCCGAACGCTGATGAC } \\
\text { R: CCTCCAGACTTGACTACACCAC }\end{array}$ & 52 & 7 & $108-174$ & 0.094 & 0.205 & 0.201 & 0.113 & 1.121 & $0.552 *$ & MF693823 \\
\hline Bih44 & $\begin{array}{l}(\mathrm{GT})_{5} \text { TAACACAG } \\
(\mathrm{GT})_{8}\end{array}$ & $\begin{array}{l}\text { F: TCCTCTCATAGCGCTCTCTTC } \\
\text { R: GCTTCACGTTACACCTGGAC }\end{array}$ & 48 & 6 & $138-166$ & 0.500 & 0.730 & 0.693 & 0.510 & 0.429 & $0.329^{*}$ & MF693824 \\
\hline Bih45 & $(\mathrm{GA})_{6}(\mathrm{GT})_{14}$ & $\begin{array}{l}\text { F: CCGGATCAGCAGAACACAG } \\
\text { R: CAGGTCAACTACAACGACACC }\end{array}$ & 60 & 8 & $207-235$ & 0.438 & 0.750 & 0.721 & 0.551 & 0.414 & $0.429 *$ & MF693825 \\
\hline Bih47 & $(\mathrm{TTAT})_{5}(\mathrm{CT})_{9}$ & $\begin{array}{l}\text { F: CTCCTCCGATACCTGTGAACTC } \\
\text { R: CCAGCAGATACACAACACAGC }\end{array}$ & 52 & 7 & $126-160$ & 0.281 & 0.571 & 0.540 & 0.363 & 0.595 & $0.518^{*}$ & MF693826 \\
\hline Bih73 & $(\mathrm{GT})_{17}$ & $\begin{array}{l}\text { F: CGCAAGCTTATGGTGAATCAG } \\
\text { R: CAATGCACGTCATTCTGC }\end{array}$ & 48 & 9 & $220-268$ & 0.188 & 0.427 & 0.416 & 0.267 & 0.779 & $0.571^{*}$ & MF693827 \\
\hline Bih76 & $(\mathrm{AC})_{19}$ & $\begin{array}{l}\text { F: CTGCATTACTCGGTAGGTTATATG } \\
\text { R: CCTGTGCAATTAAGGAACTGTG }\end{array}$ & 52 & 10 & $164-190$ & 0.500 & 0.806 & 0.779 & 0.619 & 0.368 & $0.393^{*}$ & MF693828 \\
\hline Bih77 & $\begin{array}{l}(\mathrm{AC})_{8}(\mathrm{TG})_{2} \\
\text { TTA }(\mathrm{AC})_{5}\end{array}$ & $\begin{array}{l}\text { F: TGCCAACTTCATTAGAGCTTACAG } \\
\text { R: CAGTTATATTCCTCACCTGATAGGAC }\end{array}$ & 52 & 6 & $182-192$ & 0.469 & 0.755 & 0.721 & 0.543 & 0.409 & $0.392 *$ & MF693829 \\
\hline All loci & & & & 12.8 & & 0.442 & 0.709 & 0.686 & 0.999 & $4.7 \times 10^{-6}$ & $0.367^{*}$ & \\
\hline
\end{tabular}


Tab. 2. Cross-amplification of the 15 polymorphic loci in four small Characidae species. $N$, sample size. The number in brackets represents the number of alleles. The superscript numbers are the number of individuals that successfully amplified per locus. - represents no amplification product.

\begin{tabular}{|c|c|c|c|c|c|c|c|c|c|c|c|c|c|c|c|c|}
\hline \multirow{2}{*}{ Primers Species } & \multirow{2}{*}{$N$} & \multicolumn{15}{|c|}{ Microsatellite loci } \\
\hline & & Bih13 & Bih16 & Bih19 & Bih22 & Bih26 & Bih30 & Bih31 & Bih40 & Bih42 & Bih44 & Bih45 & Bih47 & Bih73 & Bih76 & Bih77 \\
\hline & & \multicolumn{15}{|c|}{ Allele size range (bp) } \\
\hline \multirow{2}{*}{$\begin{array}{l}\text { Piabarchus } \\
\text { stramineus }\end{array}$} & \multirow{2}{*}{5} & $162-172$ & $202-216$ & $156-168$ & $208-220$ & - & $179-187$ & - & $210-238$ & - & 163 & $225-229$ & $132-164$ & $219-225$ & - & 190 \\
\hline & & $(3)^{4}$ & $(5)^{5}$ & $(4)^{5}$ & $(3)^{4}$ & - & $(2)^{5}$ & - & $(4)^{5}$ & - & $(1)^{5}$ & $(3)^{5}$ & $(6)^{5}$ & $(3)^{5}$ & - & $(1)^{5}$ \\
\hline \multirow{2}{*}{ Piabina argentea } & \multirow[b]{2}{*}{5} & 190-198 & $190-228$ & $153-175$ & $231-241$ & - & $169-221$ & - & $206-230$ & $135-159$ & $157-175$ & $197-251$ & $134-1508$ & $212-244$ & 172 & $185-199$ \\
\hline & & $(4)^{5}$ & $(5)^{5}$ & $(5)^{5}$ & $(3)^{5}$ & - & $(6)^{5}$ & - & $(5)^{5}$ & $(4)^{5}$ & $(7)^{5}$ & $(4)^{5}$ & $(4)^{5}$ & $(3)^{5}$ & $(1)^{5}$ & $(5)^{5}$ \\
\hline \multirow{2}{*}{$\begin{array}{l}\text { Serrapinnus } \\
\text { notomelas }\end{array}$} & \multirow{2}{*}{5} & - & $205-229$ & - & 212 & - & $220-240$ & - & $166-184$ & - & - & $195-223$ & - & 262 & - & - \\
\hline & & - & $(3)^{3}$ & - & $(1)^{5}$ & - & $(6)^{5}$ & - & $(4)^{3}$ & - & - & $(2)^{5}$ & - & $(1)^{5}$ & - & - \\
\hline \multirow{2}{*}{$\begin{array}{l}\text { Hyphessobrycon } \\
\text { eques }\end{array}$} & \multirow{2}{*}{5} & - & - & - & & - & $203-237$ & - & 220 & $131-133$ & - & - & - & - & - & - \\
\hline & & - & - & - & & - & $(8)^{5}$ & - & $(1)^{5}$ & $(2)^{5}$ & - & - & - & - & - & - \\
\hline
\end{tabular}

\section{Discussion}

As expected, the mixture of individuals from different streams (possibly composing different populations) resulted in deviations from the Hardy-Weinberg equilibrium (HWE) and significant $F_{I S}$ values for all loci. This is likely an indication of a Wahlund effect caused by including elements of multiple genetic units in a single panel causing excess homozygosity and significant $F_{\text {IS }}$ estimates (Freeland, 2005). From the inflated homozygosity estimates, MicroChecker suggested null alleles were present at all loci. However, it is unlikely that the excess of homozygosity was caused by null alleles as it would require all analysed loci to be affected by null alleles. Instead we favour the hypothesis that the samples were derived from genetically distinct units and the pooling of these units caused a Wahlund effect. After correction for multiple tests (sequential Bonferroni correction, $\mathrm{k}=105$ ), thirteen loci combinations showed linkage disequilibrium, the locus Bih73 was linked to loci Bih30, Bih44, Bih45, Bih47and Bih77, the locus Bih45 linked to loci Bih31, Bih40 and Bih42, the locus Bih76 linked to loci Bihl6 and Bih22, the locus Bih77 showed linkage with loci Bih 30 and Bih44, and the locus Bih44 with Bih30.

The novel microsatellite loci developed in the present study constitute a highly variable marker set suitable for genetic studies of Bryconamericus aff. iheringii. Moreover, successful cross-species amplifications of some loci indicate that these microsatellites can be used for genetic studies of other small Characidae; Piabina argentea (12 polymorphic loci) and Piabarchus stramineus (nine polymorphic loci) (Tab. 2).

\section{Acknowledgments}

We are grateful to the Universidade Estadual do Norte do Paraná (UENP) for financial and logistical support; Dr. Oscar Akio Shibatta (UEL) for his help in identifying the species studied and IBAMA (Instituto Brasileiro do Meio Ambiente e dos Recursos Renováveis), ICMBio-System (Instituto Chico Mendes-MMA) for granting permission to collect samples.

\section{References}

Agostinho AA, Thomaz SM, Gomes LC. Conservation of the biodiversity of Brazil's inland waters. Conserv Biol [serial on the internet]. 2005; 19(3):646-52. Available from: http://dx.doi. org/10.1111/j.1523-1739.2005.00701.x

Almeida FS, Fungaro MHP, Sodré LMK. RAPD and isoenzyme analysis of genetic variability in three allied species of catfish (Siluriformes: Pimelodidae) from the Tibagi River, Brazil. J Zool [serial on the internet]. 2001; 253(1):113-20. Available from: 10.1017/S0952836901000103

Billotte N, Lagoda PJL, Risterucci AM, Baurens FC. Microsatellite enriched libraries: applied methodology for the development of SSR markers in tropical crops. Fruits. 1999; 54(4):277-88.

Botstein D, White LR, Skolnick M, Davis RW. Construction of a genetic linkage map in man using restriction fragment length polymorphisms. Am J Hum Genet. 1980; 32(3):314-31.

Britski HA, Sato Y, Rosa ABS. Manual de Identificação de Peixes da Região de Três Marias, com chave de identificação para os peixes da bacia do rio São Francisco. Brasília: CODEVASF; 1988.

Castro RMC. Evolução da ictiofauna de riachos sul-americanos: padrões gerais e possíveis causais. In: Caramaschi EP, Mazzoni R, Peres-Neto PR editors. Ecologia de Peixes de Riachos. Rio de Janeiro: PPGE-UFRJ; 1999. p.139-155.

Castro RMC, Casatti L, Santos HF, Ferreira KM, Ribeiro AC, Benine RC, Dardis GZP, Melo ALA, Stopiglia R, Abreu TX, Bockmann FA, Carvalho M, Gibran FZ, Lima FCT. Estrutura e composição da ictiofauna de riachos do Rio Paranapanema, sudeste e sul do Brasil. Biota Neotrop [serial on the Internet]. 2003; 3(1):13-21. Available from: http://dx.doi.org/10.1590/S1676-06032003000100007

Cetra M, Mattox GMT, Ferreira FC, Guinato RB, Silva FV, Pedrosa M. Headwater stream fish fauna from the Upper Paranapanema River basin. Biota Neotrop [serial on the Internet]. 2016; 16(3). Available from: http://dx.doi.org/10.1590/1676-0611-BN-2015-0145

Costa ADA, Ferreira DG, Silva WF, Zanatta AS, Shibatta, AO, Galindo BA. Fishes (Osteichthyes: Actinopterygii) from the Penacho stream, upper Paraná River basin, Paraná State, Brazil. Check List [serial on the Internet]. 2013; 9(3):519-23. Available from: http://dx.doi.org/10.15560/9.3.519 
Dudgeon D, Arthington AH, Gessner MO, Kawabata ZI, Knowler DJ, Lévêque C, Naiman RJ, Prieur-Richard AH, Soto D, Stiassny MLJ, Sullivan CA. Freshwater biodiversity: importance, threats, status and conservation challenges. Biol Rev [serial on the Internet]. 2006; 81(2):163-82. Available from: http://dx.doi. org/10.1017/S1464793105006950

Excoffier L, Lischer HEL. Arlequin suite version 3.5: A new series of programs to perform population genetics analyses under Linux and Windows. Mol Ecol Resour [serial on the Internet]. 2010; 10(3):564-07. Available from: http://dx.doi.org/10.1111/ j.1755-0998.2010.02847.x

Ferreira DG, Galindo BA, Alves AN, Almeida FS, Ruas CF, Sofia $\mathrm{SH}$. Development and characterization of 14 microsatellite loci in the Neotropical fish Geophagus brasiliensis (Perciformes, Cichlidae). J Fish Biol [serial on the internet]. 2013; 83(5):143008. Available: doi:10.1111/jfb.12227

Ferreira DG, Lima SC, Frantine-Silva W, Silva JF, ApolinárioSilva C, Sofia SH, Carvalho S, Galindo BA. Fine-scale genetic structure patterns in two freshwater fish species, Geophagus brasiliensis (Osteichthyes, Cichlidae) and Astyanax altiparanae (Osteichthyes, Characidae) throughout a Neotropical stream. Genet Mol Res [serial on the Internet]. 2016; 15(4). Available from: http://dx.doi.org/10.4238/gmr15048124

Freeland JR. Molecular Ecology. Chichester (UK): J Wiley; 2005.

Graça WJ, Pavanelli CS. Peixes da planície de inundação do alto rio Paraná e áreas adjacentes. Maringá: EDUEM; 2007.

Hahn NS, Adrian IF, Fugi R, Almeida VLL. Ecologia trófica. In: Vazzoler AEAM, Agostinho AA, Hahn NS, editores. A planície de inundação do alto rio Paraná: aspectos físicos, biológicos e socioeconômicos. Maringá: EDUEM; 1997. p.209-228.

Hall TA. BioEdit: a user-friendly biological sequence alignment editor and analysis program for Windows 95/98/NT. Nucleic Acids Symp Sers. 1999; 41(41):953-08.

Langeani F, Lucena ZMS, Pedrini JL, Tarelho-Pereira FJ. Bryconamericus turiuba, a new species from the upper rio Paraná system (Ostariophysi: Characiformes). Copeia. 2005; 2005(2):386-92.

Langeani F, Castro RMC, Oyakawa OT, Shibatta OA, Pavanelli CS, Casatti L. Diversidade da ictiofauna do Alto Rio Paraná: composição atual e perspectives futures. Biota Neotrop. 2007; 7(3):181-97.

Lima FCT, Malabarba LR, Buckup PA, Silva JFP, Vari RP, Harold A, Benine R, Oyakawa OT, Pavanelli CS, Menezes NA, Lucena CAS, Malabarba MC, Lucena ZM, Reis RE, Langeani F, Cassati L, Bertaco V, Moreira C, Lucinda PHF. Genera Incertae Sedis in Characidae. In: Reis RE., Kullander SO, Ferraris Jr CJ, editors. Check List of the Freshwater Fishes of South and Central America. Porto Alegre: EDIPUCRS; 2003. p.106-169.

Lowe-McConnell RH. Estudos ecológicos de comunidades de peixes tropicais. São Paulo: EDUSP; 1999.

Marshall TC, Slate J, Kruuk LE, Pemberton JM. Statistical confidence for likelihood-based paternity inference in natural populations. Mol Ecol. 1998; 7(5):639-55.

Paetkau D, Calvert W, Stirling I, Strobeck C. Microsatellite analysis of population structure in Canadian polar bears. Mol Ecol. 1995; 4(3):347-54.
Raymond M, Rousset F. GENEPOP (version 1.2): population genetics software for exact tests and ecumenicism. J Hered. 1995; 83(3):248-49.

Rice WR. Analyzing tables of statistical tests. Evolution. 1989; 43(1):223-25.

Rousset F. GENEPOP'007: a complete re-implementation the GENEPOP software for Windows and Linux. Mol Ecol Resour [serial on the internet]. 2008; 8(1):103-06. Available from: http://dx.doi.org/10.1111/j.1471-8286.2007.01931.x

Rozen S, Skaletsky HJ. Primer 3 on the www for general users and for biologist programmers. In: Krawetz S, Misener S, editors. Bioinformatics Methods and Protocols: Methods in Molecular Biology. Totowa (NJ): Humana Press; 2000. p.365-386.

Schuelke M. An economic method for the fluorescent labelling of PCR fragments. Nat Biotechnol [serial on the internet]. 2000; 18(2):233-34. Available from: http://dx.doi.org/10.1038/72708

Sofia SH, Galindo BA, Paula FM, Sodré LMK, Martinez CBR. Genetic diversity of Hypostomus ancistroides (Teleostei, Loricariidae) from an urban stream. Genet Mol Biol [serial on the internet]. 2008; 31(1):317-23. Available from: http://dx.doi. org/10.1590/S1415-47572008000200027

Sofia SH, Silva CRM, Galindo BA, Almeida FS, Sodré LMK, Martinez CBR. Population genetic structure of Astyanax scabripinis (Teleostei, Characidae) from an urban stream. Hydrobiologia [serial on the internet]. 2006; 553(1):245-54. Available from: http://dx.doi.org/10.1007/s10750-005-1106-4

Vallone PM, Butler JM. AutoDimer: a screening tool for primer-dimer and hairpin structures. BioTechniques. 2004; 37(2):226-31.

Van Oosterhout C, Hutchinson WF, Wills DPM, Shipley P. MICRO-CHECKER: software for identifying and correcting genotyping errors in microsatellite data. Mol Ecol Notes [serial on the internet]. 2004; 4(3):535-08. Available from: http:// dx.doi.org/10.1111/j.1471-8286.2004.00684.x

Vari RP, Siebert DJ. A new unusually sexually dimorphic species of Bryconamericus (Pisces: Ostariophysi: Characidae) from the Peruvian Amazon. Proc Biol Soc Wash. 1990; 103(3):516-24.

Wagner HW, Sefc KM. IDENTITY 1.0. Vienna: Centre for Applied Genetics, University of Agricultural Sciences [serial on the Internet] 1999. Available from: http://www.boku.ac.at/zag/ forsch/ identity.htm

Weir BS. Genetic Data Analysis II. Methods for Discrete Population Genetic Data. Sunderland (MA): Sinauer Associates Inc. Publishers; 1996.

Winemiller KO, Agostinho AA, Caramaschi EP. Fish ecology in tropical streams. In: Dudgeon D, editor. Tropical stream ecology. Amsterdam: Academic Press; 2008. p.1073-1046. 
